Cinémas

Revue d'études cinématographiques

Journal of Film Studies

\title{
Le Voir-dire
}

\section{Max Vernet}

Volume 4, numéro 1, automne 1993

\section{Écrit/Écran}

URI : https://id.erudit.org/iderudit/1000109ar

DOI : https://doi.org/10.7202/1000109ar

Aller au sommaire du numéro

\section{Éditeur(s)}

Cinémas

ISSN

1181-6945 (imprimé)

1705-6500 (numérique)

Découvrir la revue

\section{Citer cet article}

Vernet, M. (1993). Le Voir-dire. Cinémas, 4(1), 35-47. https://doi.org/10.7202/1000109ar

\section{Résumé de l'article}

La question est celle du rapport du théâtre, du récit et du film à l'intériorité, question qui peut se poser à partir de trois textes : le Tartuffe de Molière, l'Onuphre de La Bruyère, et le Tartüff de Murnau. L'hypocrite est en effet pour le théâtre, art de l'extériorité et des relations, une boîte noire; il est exemplaire de la difficulté qu'a ce genre à voir l'intérieur, difficulté contre laquelle il invente la passion : mouvement visible en surface du mouvement de l'âme. Le récit classique, lui, dans sa forme romanesque, recueille les passions en un " caractère ", qui est l'intériorité dite au service de l'enchaînement du récit progressant pas oscillation simple de l'intérieur (le caractère des personnages) à l'extérieur (leurs actes). L'examen de quelques plans de Tartüff peut montrer que le cinéma pourrait être interprété comme la synthèse sur ce point du théâtre et du récit, comme le lieu moderne de la tautologie passionnelle. Mais que, sur deux plans figés indécidables, il est également possible de croire que le cinéma, accueillant en lui sa négation, aurait su montrer l'événement.
Ce document est protégé par la loi sur le droit d'auteur. L'utilisation des services d'Érudit (y compris la reproduction) est assujettie à sa politique d'utilisation que vous pouvez consulter en ligne.

https://apropos.erudit.org/fr/usagers/politique-dutilisation/ 


\title{
Le Voir-dire
}

\section{Max Vernet}

\begin{abstract}
RÉSUMÉ
La question est celle du rapport du théâtre, du récit et du film à l'intériorité, question qui peut se poser à partir de trois textes : le Tartuffe de Molière, l'Onuphre de La Bruyère, et le Tartüff de Murnau. L'hypocrite est en effet pour le théâtre, art de l'extériorité et des relations, une boîte noire; il est exemplaire de la difficulté qu'a ce genre à voir l'intérieur, difficulté contre laquelle il invente la passion : mouvement visible en surface du mouvement de l'âme. Le récit classique, lui, dans sa forme romanesque, recueille les passions en un "caractère", qui est l'intériorité dite au service de l'enchaînement du récit progressant pas oscillation simple de l'intérieur (le caractère des personnages) à l'extérieur (leurs actes). L'examen de quelques plans de Tartüff peut montrer que le cinéma pourrait être interprété comme la synthèse sur ce point du théâtre et du récit, comme le lieu moderne de la tautologie passionnelle. Mais que, sur deux plans figés indécidables, il est également possible de croire que le cinéma, accueillant en lui sa négation, aurait su montrer l'événement.
\end{abstract}

\begin{abstract}
The question of the relationship of theatre, story and film to interiority is posed on the basis of three texts : Molière's Tartuffe, La Bruyère's Onuphre, and Murnau's Tartüff. For the theatre, the hypocrite is in fact an art of exteriority and of relationships, a black box, an example of how hard it is for this genre to see the interior. To counter this difficulty, it invents passion a surface movement making visible the movement of the soul. For the classic story in its novelistic form, the passions are incorporated in a character, an interiority said to serve the progress of the plot, moving it forward through a simple oscillation between the interior (the
\end{abstract}


personality of the characters) and the exterior (their actions). An examination of a number of shots from Tartüff reveals that in this regard cinema may be considered a synthesis of theatre and story, the modern site of a passionate tautology. But two frozen undecidable shots also allow us to believe that the cinema, by accepting its own negation, has found a way to show the event.

La vérité se présente comme une chute, comme un glissement et une erreur : ce que veut dire en latin lapsus. L'événement ouvre un espace et un temps de vertige, il n'est pas rattaché à son contexte ou à son environnement perceptif.

Lyotard

Discours, Figure, p. 135.

D'abord, l'explication du titre. Il joue en fait sur deux sens de «voir»: l'un, moderne, infinitif; l'autre, ancien, celle de l'ancienne orthographe de vrai : dire-vrai ${ }^{1}$. Et dans le langage juridique, une «assignation de voir dire et ordonner» est une injonction d'être présent lorsqu'est proclamé un ordre. La conjonction du vrai et du voir par la présence balise ainsi toute la pensée moderne, depuis la révolution scientifique, et doit bien avoir quelque chose à voir avec le théâtre classique, et peut-être avec le cinéma.

Le plus court, c'est de donner d'abord les mots clés; ils serviront comme à sauter de pierre en pierre dans le cours du texte et, finalement, à voir d'abord ce que j'ai à dire. Note : en bonne langue du XVIIe siècle "d'abord» veut dire dès l'abord, immédiatement. Celui qui dans le dénouement de Tartuffe voit d'abord, c'est le Roi, qui n'est jamais trompé par les artifices de l'hypocrite.

Mots clés donc : Tartuffe. Théâtre, récit, cinéma. Molière, La Bruyère, Murnau. Vision, vérité. Écart, secret, différence, individu. Passion, inconscient. Événement.

Corpus : le Tartuffe de Molière, 1669. L'Onuphre de La Bruyère, qui est le 24 du chapitre "De la Mode», dans la sixième édition, 1691. Le Tartüff de Murnau, 1925; plus particulièrement, une séquence de la rencontre de Tartüff et d'Elmire dans le boudoir, rencontre organisée par Elmire et qui doit servir à démasquer Tartüff aux yeux d'Orgon caché; la manigance échouera lorsque in extremis Tartüff apercevra, déformé par la théière, le reflet du mari. J'ajoute, pour m'en débarrasser aussitôt, que la situation globale d'énonciation est 
chez Murnau celle d'une persuasion, un peu comparable à celle du théâtre dans le théâtre de Hamlet : une version filmée du Tartuffe de Molière est montrée à une gouvernante hypocrite et à sa victime. Il s'agit donc bien du Tartuffe de Molière, mais, c'est important pour la suite du texte, ce n'est pas du théâtre filmé 2 .

On se souvient qu'au début de Gödel, Escher, Bach, Hofstadter imagine un enregistrement sonore tel que, mis sur une platine, il détruirait l'appareil de son qui le reproduirait. On pourrait dire que Tartuffe est, pour le théâtre, ce logiciel assassin. Non tant que Tartuffe, le personnage, ne soit pas visible, qu'on ne puisse le montrer au théâtre (c'est pourtant bien vrai, c'est ce qu'a rencontré Molière, qui passe deux actes entiers à préparer l'entrée de l'Hypocrite). C'est qu'il n'est pas dis-cernable, que le regard ne peut séparer la copie du modèle, le théâtre du réel, bouclant autour de Molière un paradoxe semblable à celui du menteur : Molière dit que Tartuffe est un hypocrite, or Molière est un acteur. Si on ne peut voir la différence dans ce théâtre, c'est qu'on ne peut voir la différence «du théâtre», à cause de cette «idée de derrière» chez Molière que tout est relations. Ou plutôt que ce que le théâtre dit le plus aisément, c'est un monde d'interactions. Des personnages se disent en scène non pas par l'adéquation de leurs gestes (et quand je dis gestes, je leur assimile la mimique, parlant comme je fais du théâtre du XVIIe) à un intérieur à jamais hors représentation, mais par l'ensemble des relations qu'ils entretiennent les uns avec les autres. C'est le clou qu'enfonce Molière avec une grande constance : l'individualité est le $s e$-cret, ce que quelqu'un $a b$-strait du domaine public. Argent de l'avare, femme d'Arnolphe, prestige de classe de Dom Juan, attraits de Célimène, tout ce qui garantit la différence est en fait quelque chose qui a son existence légitime dans la circulation, la relation, et qui a été confisqué et immobilisé. L'individualité est un espace $s e$-cret, la différence est un écart : maison gardée d'Arnolphe, jardin d'Harpagon, chambre de Célimène. Le théâtre vit d'espace et de relations, ce qui est une tautologie. Les illégitimes vivent de distraction, détournement, immobilisation : au-dehors, la science du théâtre est la politique, qui théorise l'ensemble des relations. Tartuffe n'est visible que du roi (comme on dit du haut de l'Arc de triomphe) vers qui convergent toutes les relations. Tartuffe est la plus grande menace, parce qu'il dis-trait, confisque à son profit la relation de connaissance et la représentation même : comme si le paysage savait qu'il va être peint, et se disposait en conséquence. J'aime beaucoup l'idée : une montagne Sainte- 
Victoire hypocrite se couvrant d'événements colorés pour monsieur Cézanne, et retournant à sa bêtise grise lorsqu'il n'est plus là; et combien de tempétueuses marines sont-elles le fait d'océans vaniteux?

Tartuffe est la plus grande menace pour le théâtre parce qu'il faudrait savoir ce qu'est le représenté juste avant la représentation, et qu'il faudrait pour cela sortir du théâtre et saisir l'événement qu'est la représentation; et Jouvet trichait : Tartuffe - «apercevant Dorine» - remontait trois marches d'escalier et redescendait d'un tout autre air.

La rançon de ce théâtre est la paranoïa : «montre-moi tes mains»... «les autres». "Qui m'assure, clame Alceste, qu'au même instant vous n'en dites pas autant à d'autres?» Il faudrait voir derrière, occuper tout l'espace qui par définition n'est vu qu'encadré par le manteau d'Ârlequin, nié par les coulisses, aplati par le point de vue unique du spectateur. Voir Tartuffe dans tout le continuum : dans sa chambre, avec sa fripouille de valet; voir la longue suite «d'actions toutes noires» qui permettent enfin de le condamner. Comme le dieu qui vit Sosie, seul dans sa tente, bouffer un jambon. La menace que fait planer Tartuffe appelle le récit comme son antidote : un diable boiteux est autrement efficace, pour voir les intérieurs, qu'un roi. Le récit serait la solution du théâtre.

C'est bien ce que dit La Bruyère à Molière, à qui il fait la leçon:

Il ne dit point ma haire et ma discipline, au contraire, il passerait pour ce qu'il est, pour un hypocrite, et il veut passer pour ce qu'il n'est pas, pour un homme dévot; il est vrai qu'il fait en sorte que l'on croit, sans qu'il le dise, qu'il porte une haire et qu'il se donne la discipline. Il y a quelques livres répandus dans sa chambre indifféremment, ouvrez-les : c'est Le Combat spirituel, Le Chrétien intérieur, et L'Année sainte; d'autres livres sont sous la clef. S'il marche par la ville et qu'il découvre de loin un homme devant qui il est nécessaire qu'il soit dévot, les yeux baissés, la démarche lente et modeste, l'air recueilli lui sont familiers, il joue son rôle. S'il entre dans une église, il observe d'abord de qui il peut être vu, et selon la découverte qu'il vient de faire il se met à genoux et prie, ou il ne songe ni à se mettre à genoux ni à prier (...). Il entre une autre fois dans un lieu saint, perce la foule, choisit un endroit pour se recueillir, ou tout le monde voit qu'il s'humilie (...). Il évite une église déserte et solitaire, où il pourrait entendre deux messes de suite, le sermon, vêpres et complies, tout cela entre Dieu et lui, et sans que personne lui en sût gré; il aime la paroisse, il fréquente les temples où se fait un grand concours, on n'y manque point son coup, on y est vu.

Texte retors, dans lequel je ne vais pas craindre d'avancer à petits pas. 
D'abord pour dire que c'est un texte manifestement polémique, au sens qu'il s'annonce comme la réponse à un autre texte. Tartuffe, dit La Bruyère, n'est pas vrai; ou du moins Tartuffe est un débutant en matière d'hypocrisie : il ne durerait pas dix jours. Mon commentaire sera que l'Onuphre n'est pas tant un meilleur Tartuffe qu'un Tartuffe narré, et que l'opposition est entre deux genres, le récit et le théâtre, et non entre deux individus. Et que la question à retourner à La Bruyère, c'est : comment Onuphre serait-il réel, si Tartuffe n'existait pas?

Il est clair en fait que ce que reproche La Bruyère à Molière, c'est que son Tartuffe est visible, alors que le jeu du véritable hypocrite serait d'être couleur de muraille, couleur de tout le monde. La raison pour laquelle Tartuffe serait dans le monde réel immédiatement découvert, dit-il («il passerait pour ce qu'il est»), c'est qu'il en fait trop; et La Bruyère continue en donnant la vérité en récit du véritable hypocrite. Il lui reproche, c'est évident, son côté théâtral : à quoi on imagine assez bien Molière, prolongeant la préface de son œuvre, lui répondant : que devaisje faire (...)? C'est donc au dépassement de Tartuffe par Onuphre, du théâtre par le récit, que je vais m'intéresser : La Bruyère n'a aucun doute sur l'efficacité du récit pour ce faire.

Je note d'abord la capacité du récit à parcourir tout l'espace. Cette chambre de l'hypocrite d'où il sort et où il rentre sans qu'on puisse au théâtre la voir (pas plus que celles de Célimène et d'Arnolphe, pas plus que le jardin d'Harpagon), nous y voici conviés à une fouille : «Il y a quelques livres (...), ouvrez-les».

Espace qui contient l'intériorité même d'Onuphre, traversée sans qu'il y paraisse : «(...) il passerait pour ce qu'il est, (...) et il veut passer pour ce qu'il n'est pas (...)» Voilà qui va à merveille, dit Molière, et n'est-il pas beaucoup plus simple d'écrire «il veut passer» que de s'acharner à le faire voir deux grands actes durant? On peut aussi par le récit se permettre bien mieux : on peut dire ce qui ne se passe pas, ce que le protagoniste évite de faire («Il évite une église déserte et solitaire»), et ce qui pourrait se passer («où il pourrait entendre deux messes de suite»). Les contrafactuels sont d'un efficace merveilleux pour décrire la non-coïncidence entre les actes et la pensée, et le récit passe aisément la frontière du crâne : diable boiteux et omniscient.

Mais il y a aussi une manipulation du temps qui peut-être n'apparaît pas à première vue, si on ne se souvient qu'Onuphre est un "caractère» et non un héros de roman. Autrement dit, sa vérité est celle du vraisemblable, il est ce que fait vraisemblablement l'hypocrite, au sens collectif que nous 
appelons un peu vite le «type» de l'hypocrite. Autrement et plus clairement dit, la suite des actions décrites comme étant le fait d'Onuphre ( «S'il marche par la ville (...) S'il entre dans une église (...) S'il évite une église (...)») n'est pas une séquence le long d'une durée, c'est une collection d'exempla de ce que peut faire un hypocrite.

(Ce qui vend la mèche, c'est le «ou» dans : «(...) selon la découverte qu'il vient de faire, il se met à genoux (...) "ou" il ne songe ni à prier (...).» Le récit classique, art du temps, est le lieu des successifs, des éléments enchaînés par «et»; comme dirait Figaro, qu'il y ait «et» ou bien «ou» fait toute la différence; et si Robbe-Grillet a posé tant de problèmes, c'est bien qu'on ne peut savoir - au cinéma comme dans l'écrit - quand il dit «et»et quand il dit «ou»).

Chaque acte hypocrite, que La Bruyère veut invisible, ne devient visible comme instance d'hypocrisie que par l'accumulation, non pas d'«actions toutes noires» faites par un seul individu, mais par l'observation du moraliste qui abstrait de la conduite du captateur d'héritage, du parasite, du calomniateur, du faux dévot ce qu'elles ont de commun. Onuphre n'est donc pas un personnage de roman, mais un "caractère», un chapitre d'une sorte de manuel qui pourra servir aux lecteurs de La Bruyère à reconnaître un hypocrite. Nous ne sommes donc pas ici dans la représentation, dans le présent de l'indicatif de l'événement présenté, mais dans le présent de la loi scientifique. Et c'est ce savoir abstrait qui permet d'épingler l'hypocrite réel, et aussi Tartuffe, le pauvre homme : à qui possède ce savoir, celui-ci paraît évidemment bien théâtral. Pourtant, ajoutant un épisode à l'histoire de la retraite des genres persuasifs devant le narratif, La Bruyère narre, dissimulant le savoir abstrait derrière l'enchaînement du récit, le caractère (ce qui permet de reconnaître) derrière le caractère (ce qui, dans le roman, assure la constance de l'identité). Vu de loin et de dos, comme nous le faisons, il semble bien difficile de séparer l'un de l'autre : c'est bien ainsi pourtant, je crois, que naît au XVIIe siècle la différence. Elle ne se donne plus comme espèce sous un genre, elle sera désormais ce qui se décrira à partir de parcours spatiotemporels soigneusement impliqués, enveloppés à l'intérieur du nom propre de l'éponyme : l'identité est ce qui le met à part «et» ce qui est constant. Ce qu'un balayage de l'espace révélera de différent, et qu'un balayage du temps confirmera dans sa différence; le roman classique est bâti sur cette oscillation simple de l'invisible au visible. 
Le théâtre bute sur la différence comme sur sa mort; donnant des personnages «en pied» dans leurs relations complètes, il est dans la tradition classique française sans extérieur : sa loi est celle de l'ici-maintenant et de la surface. La réserve, la thésaurisation, l'identité comme creux dans les relations s'y disent très mal. Je crois que celui de Molière, où liberté rime avec écart («Et trouver loin d'ici un endroit écarté / Où d'être homme d'honneur on ait la liberté» 3 ), vit la différence comme erreur du politique : écart, au sens aussi d'écart de conduite. En ce sens, le récit affirme par-dessus le théâtre la conquête de l'invisible, et donne la différence non plus comme ce qui troue le politique mais comme ce qui, à l'intérieur d'une limite, s'enlève sur le fond d'une science de l'Homme, transgresse un vraisemblable, auquel finalement il sera reversé. Cette science a longtemps porté dans le roman le nom de psychologie. Il va de soi que ceci peut être accepté comme un progrès : il me semble en fait peu probable que l'Homme classique ait pu se former sans ce récit. Mais que l'on appelle «point de vue omniscient» l'infinie mobilité du récit tendrait à prouver que l'on voit cet avantage du récit, comme l'a fait $\mathrm{La}$ Bruyère, comme une solution aux problèmes du théâtre : solution technique qui remédierait aux défauts d'une forme artistique dont la «vue» était partielle et inadéquate à son objet. En fait, bien sûr, le récit ne voit rien du tout : il dit voir pour faire croire qu'il dit vrai, parce que dans un système où la vérité est dévoilement, le voir est toujours la limite et l'achèvement du dire. Sourde revanche du théâtre.

Deuxième partie : où mes lecteurs, qui ont perçu le balancement de voir dire à dire voir, me voient venir avec ma synthèse qui serait le cinéma. Bon, faisons comme. Hypocrite.

Le classicisme français invente la passion; peut-être pressé dans ses derniers retranchements par le récit, le classicisme invente une théorie de l'Homme telle que, son corps lui échappant, ce qui lui échappe n'échappe pas aux autres. Car la passion est mouvement, agitation plus précisément, c'est-à-dire mouvement non contrôlé par la raison. Hors contrôle, elle est l'anti-Tartuffe; productrice de signes irrécusables que l'on ne saurait supprimer; signes-indices, qui sont à la passion ce que la fumée est au feu, mouvement visible produit par un autre mouvement caché. On ne saurait donc cacher la passion, et celleci est ainsi au fondement d'une sémiotique. Le tekmérion classique est la rougeur : elle fonde cette science de la femme qui est la grande menace qui poursuit Mme de Clèves :

M. de Clèves se trouvait heureux sans être néanmoins entièrement content. Il voyait avec beaucoup de peine que les sentiments de 
Mlle de Chartres ne passaient pas ceux de l'estime et de la reconnaissance (...).

- Vous ne sauriez douter, reprit-elle, que je n'aie de la joie de vous voir, et je rougis si souvent en vous voyant que vous ne sauriez douter aussi que votre vue ne me donne du trouble.

- Je ne me trompe pas à votre rougeur, répondit-il; c'est un sentiment de modestie, et non pas un mouvement de votre cœur (...) (pp. 258-259).

(On pourrait dire entre parenthèses que la psychologie n'est pas plutôt née qu'elle est récusée : si c'est ça la science de l'Homme, dit à peu près Mme de Clèves, je me retire au couvent; et sa sœur en religion : «Suis-je obligée de vous rendre compte de tous mes mouvements? $\left.{ }^{4} »\right)$

Mais plus généralement, la passion fonde cette théâtralisation par laquelle on se plaît parfois à définir l'âge classique. Pourtant il n'est pas difficile de s'apercevoir que dans le théâtre que j'ai défini plus haut comme la présentation ( $=$ montrer au présent) de relations, cette psychologie a peu de place. La loi du théâtre est que les personnages y sont en pied, et ont toujours pour le spectateur la même dimension relative; aucun dispositif ne permet de prélever sur le visage de Phèdre la rougeur et la pâleur qui se succèdent à la vue du fils de l'Amazone. Ce que nous ne saurions voir, un récit nous l'apprend. On sait par contre que le geste suppléait à ce que, enfants du cinéma, nous nous attendons à voir sur les visages : la surprise, la peur, etc. avaient leurs gestes. Mais voilà, le geste est toujours contrôlable, donc suspect d'être contrôlé. Le récit dira : elle ne put retenir un geste de surprise; le théâtre montrera un geste de surprise «et» un geste de dissimulation du geste de surprise. Tout le dispositif fait que ces gestes peuvent être volontaires : qu'ils soient par le personnage retenus ou non, indices ou symboles, dépendra hélas de l'interprétation du spectateur, c'est-à-dire du contexte, c'est-àdire de la totalité des relations en scène. Et pour une Dorine, combien de Pernelles?

Il faudra donc aller prélever sur les visages les expressions, les troubles que le récit traquait si bien mais représentait si mal, traduisant en langage, domaine par excellence de l'arbitraire, ce qui en fait le fondait en vérité : l'indice passionnel qui est enfin la garantie du voir dire. On peut prendre toute cette littérature classique sur la parfaite amitié, sur les incertitudes de l'amour, comme une interrogation sur ce qui fonde le signe, sur une sémiotique générale née de la nécessité politique de vivre en société. La Cour en est le microcosme expérimental : 
Il y avait tant d'intérêts et tant de cabales différentes, et les dames y avaient tant de part que l'amour était toujours mêlé aux affaires et les affaires à l'amour (p. 252).

Le cinéma, nous y voilà, serait ce rêve du théâtre racinien; voir Phèdre au plus près de Phèdre, voir l'émotion que le récit ne savait que dire, être un récit vu. Dire l'intérieur à coup sûr, en voyant une surface qui n'en est pas une et traduit la profondeur du mouvement pour le spectateur exactement comme chez Descartes, pour l'âme : par contiguiité. Voir vrai, voir voir.

Troisième partie : où la synthèse n'est pas celle qu'on pense. Car il est possible que ne soit pas ce qui se passe chez Murnau. Il y a évidemment un cinéma qui serait du récit vu, et on imagine très bien la complicité du cinéma et du récit de La Bruyère, qui semble avoir préparé les séquences et les plans :

\begin{abstract}
Il y a quelques livres répandus dans sa chambre [plan moyen] indifféremment, ouvrez-les : c'est Le Combat spirituel, Le Chrétien intérieur, et L'Année sainte [insert sur les couvertures des livres]; d'autres livres [plan rapproché] sont sous la clef [insert sur le clef]. S'il marche par la ville [plan moyen] et qu'il découvre [gros plan des yeux] de loin [plan lointain] un homme [plan moyen] devant qui il est nécessaire qu'il soit dévot, les yeux baissés [contrechamp, gros plan], la démarche lente et modeste [plan moyen], l'air recueilli [gros plan] lui sont familiers, il joue son rôle. S'il entre dans une église [plan lointain], il observe d'abord [gros plan] de qui il peut être vu [panoramique de l'intérieur de l'église], et selon la découverte qu'il vient de faire [plan rapproché] il se met à genoux et prie [contrechamp, plan moyen] (...).
\end{abstract}

Ici le cinéma et le récit sont bien plus complices que ne pourraient l'être le théâtre et le cinéma, justement à cause de la mobilité de la caméra. Mobilité de la caméra, qui pourtant s'époumone souvent à suivre la mobilité diabolique et boiteuse du récit. Des bielles de train s'agitent furieusement pour dire : «le lendemain à Vienne»; des journaux tournoient pour dire : l'année suivante. Et combien de caméras angéliques à l'œil blindé crèvent doucement les toits, les murs et les fenêtres? Ce qui semblerait indiquer que la caméra ne s'assujettit que difficilement au récit et que le point de vue romanesque est effectivement un point (sans dimension), mais pas de vue. Au passage, notons que l'enchaînement champ contrechamp, jusque dans sa dénomination, contient la spécificité du cinéma par rapport au théâtre (l'absence d'envers, de dos) et son mensonge fondamental, qui prétend que les deux espaces du champ et du contrechamp sont complémentaires sans reste, alors que c'est ce 
qui fonde l'absence d'un espace de la caméra, devenue, effectivement «point» de vue.

Je voudrais maintenant extraire de ce pseudo-texte filmé à partir de La Bruyère la pseudo-séquence de la découverte; où l'on voit Onuphre voir : "S'il marche par la ville et qu'il découvre (...)» Au cinéma, la succession de [gros plan sur les yeux de $x$ ] et de [plan $p$ sur quelque chose] dit : $x$ voit quelque chose. En prolongeant un peu, et en ajoutant un gros plan sur le visage, on aura : $x$ voit quelque chose, et il a telle réaction. Nous ne sommes pas sortis du récit, et nous avons, en un sens nouveau, une caméra-stylo qui enchaîne classiquement action et passion, acte et «sentiment», en recueillant sur le visage l'«expression» (le beau mot : la pression vers l'extérieur de l'intériorité qui n'a rien de plus pressé que d'aller se dire). Autant dire qu'une telle intériorité, autre du même, contribuant en fait à enchaîner du «visible» par de l'«invisible», n'a rien d'une différence. De même qu'en mécanique classique, la somme des énergies est toujours la même et qu'on peut toujours retransformer de l'énergie cinétique en énergie potentielle, dans le roman et dans le film-récit, le caractère (la différence individuelle) est monnayable en actions, et vice versa, le caractère n'est que la somme des actes du personnage. Le dire-voir est à ce prix. Or je ne pense pas que ce soit ce qui se passe chez Murnau.

Quatrième partie, qui sera peut-être la plus courte. La séquence du film Tartüff que j'ai annoncée plus haut tient en neuf secondes, à l'intérieur de la deuxième rencontre d'Elmire et de Tartüff : Elmire, qui l'a fait venir dans son boudoir, sert une collation. Fondant en larmes, elle «avoue» à Tartüff qu'elle croit l'aimer et laisse tomber son mouchoir. Ici commencent les quatre plans qui m'occupent.

- plan 1 (4 secondes) : Plongée. Gros plan sur la jupe d'Elmire assise. En ramassant le mouchoir, celle-ci découvre jusqu'au mollet sa jambe droite, alors que le pied gauche, ramené vers l'arrière, maintient la jupe relevée. L'image est, dans la presque totalité de la dernière seconde, immobile.

- plan 2 (3 secondes) : Contrechamp. Gros plan du visage de Tartüff, qui regarde vers le bas (!), immobile. L'œil droit, peutêtre, se ferme légèrement.

- plan 3 (1 seconde) : Plongée, comme ci-dessus. Gros plan du pied découvert d'Elmire. C'est le photogramme qui termine le plan 1.

- plan 4 : Contrechamp, comme ci-dessus. Gros plan du visage de Tartüff, comme en 2, immobile. 
Il y aura aussi, comme en écho, quelques secondes plus tard, deux plans qui répètent 3 et 4 .

Lu dans le système du film-récit, la séquence donne : Tartuffe désire Elmire. Et si j'en crois la critique de cinéma, c'est justement ce montage de corps morcelés qui rompt un des contrats de base, qui est celui de la distance à la caméra. Toute transgression de ce contrat indiquerait une émotion du protagoniste, un passage dans le non-rationnel. Ce qui dirait le désir de Tartuffe, ce serait donc que le pied occupe tout le champ; on aurait là l'équivalent de la rupture de l'alexandrin chez Racine. Le gros plan dirait l'incomplétude du focalisateur interne (j'emploie ici, je crois, le terme dans son emploi narratologique balien; en fontanillien, il s'appelle l'Assistant) déjà notée par le gros plan sur le visage : le désir, rompant la perception "normale», s'adresserait à une partie seulement du corps d'autrui. Le désir serait l'incomplétude du sujet; seul le sujet rationnel, complet, s'adresserait à la totalité de la personne de l'autre; ce qui est de la bien éculée philosophie. Or j'espère que ce n'est pas ce qui se passe chez Murnau.

Je ne conteste nullement la nécessité pour l'inhabituel d'avoir à s'appuyer sur et à s'enlever contre de l'habituel. En ce sens en effet, la séquence entière «et» chacun des plans sont bien mis en place par Murnau à l'intérieur d'une séquence de conversation normale : plan d'ensemble du salon, plans moyens de Tartuffe et d'Elmire. C'est la nature de la transgression qui me paraît demander examen.

En fait, tout repose sur une interprétation, et j'aime finalement ces moments indécidables où une chose est deux choses à la fois : moment de bifurcation, singularité, inflexion de courbes, bégaiement de l'histoire, symptôme.

Tout se joue sur la décision suivante : poser que les gros plans sur le visage de Tartuffe et sur le pied d'Elmire sont ou bien des plans qui filment une immobilité, ou bien des plans figés, c'est-àdire répétant par montage le même photogramme. L'enjeı est bien sûr la transgression sur le temps (noter que le film est muet, c'est une chance; point de musique bavarde qui raconte à la place de l'image, ou de voix off, qui rétabliraient en coulisse le temps disparu; mais aussi doublement : pas de cartons non plus, contrairement au reste du film 5 ; le silence de cette séquence de film muet est oppressant).

Si Murnau filme une immobilité, alors nous ne sommes pas sortis du récit qui raconte une immobilité : Tartuffe immobile désire Elmire immobile, les deux forment un couple passionné. Le désir de Tartuffe est au fond aussi la passion d'Elmire, ce 
qu'elle subit, ce qu'elle laisse l'immobiliser, fascinée. Ce serait peut-être effectivement très allemand 1925 . Or ce n'est pas ce qui se passe chez Murnau si on a un plan figé6.

Car la transgression ici ne porte plus seulement sur le contrat cinématographique, sur les habitudes de cadrage du cinéma «art de l'espace», la transgression prend en écharpe et sectionne le cinéma art du temps. Que se passe-t-il alors "pendant» le pied d'Elmire? Justement, rien. C'est l'événement même, l'accident fondamental, la catastrophe qui atteint au cœur le récit dans sa concaténation, dans sa mobilité, et donc dans toutes les passions du monde. Et donc aussi, dans tous les syntagmes. Le plan figé dit, mais justement, il le dit avant tout dire : il y a du pied, il y a de l'œil. Pas de temps, un espace inassignable : nous reconnaissons l'inconscient, et sa manière radicale de trouer le discours. Je ne vais pas faire ici le coup de la pulsion scopique, du corps morcelé, de la femme-objet ni même du sadisme oral ou anal, préférant le silence à la psychanalyse. C'est l'événement pour Tartuffe, pris à partie, mis en parties par son propre désir et par l'hallucination d'un pied se voulant vu. Non plus une passion, une intériorité accourant pour se dire, mais un mondedésir réorganisé par d'inquiétantes lois.

Le théâtre ne peut dire ni l'intériorité ni le fétichisme; le récit ne peut dire l'événement, qui est toujours traversé par la mise en récit comme les passions sont traversées par le caractère. Pour dire le bouleversement, le récit est obligé de raconter et, ce faisant, d'assimiler au même ce qui viendrait l'interrompre. Exemple peut-être de ce qu'on peut faire de mieux en la matière, Flaubert, L'Éducation sentimentale - «Et Frédéric, béant, reconnut Sénécal» - , où le maître de l'amuïssement du discours bute sur sa propre limite et raconte la béance.

Reste le cinéma que le plan figé menace tellement qu'on n'a pas craint de dire qu'il n'est pas cinématographique 7 . En fait, on pourrait proposer qu'il est un moyen (le seul?) par lequel le cinéma peut s'affranchir du récit, son aîné.

En fait, bien sûr, rien n'est sûr. Tout cela est marqué de l'après-coup. Il y a un moment où effectivement le spectateur à qui on a depuis appris le plan figé peut, dans chacun des plans, voir un plan figé : pied, visage, pied. Mais il faut tenir compte de la lenteur constitutive de Tartüff. Chacun des plans se prolonge ou se résoud en un mouvement qui n'est perceptible qu'au ralenti : le visage de Tartüff est pris, dans les dernières secondes du plan, par un imperceptible et invincible mouvement vers le bas (comprenons : en direction du pied, pris par la gravité du péché). Et le deuxième plan du pied d'Elmire est coupé sur «un» 
photogramme où le mouvement de l'autre pied (mouvement symétrique, vers l'avant, du mouvement qu'il effectuait dans le plan précédant, celui du visage de Tartüff) est «flou», trop rapide pour la caméra et impossible à saisir en visionnement normal. Le mouvement habite donc cette séquence comme une sorte de réminiscence ou d'afterthoughts. Le plan figé l'habite comme un malaise («il a eu un malaise») du récit.

Il reste ainsi cette idée tentante où, une fois l'Homme classique et ses passions, ses récits, son théâtre disparu, au moment où apparaît un sujet étoilé entre Moi, Surmoi, et Ça, le cinéma avait pu être l'art de suspendre le bavardage de l'humanisme et d'accueillir l'événement.

Mais qui dira l'à voir vu?

Queen's University

\section{NOTES}

1 Comme dans Le Voir dit, titre de Guillaume de Machaut, que m'a montré René Gagné.

2 Voir Patrice Pavis, «Le Théâtre au cinéma : prolégomènes à une noncomparaison (à propos du Tartuffe de Murnau)», Protée vol. 17 no 1 (hiver 1989) p. 46. C'est ici le lieu de dire combien cette étude doit au séminaire qu'il a donné à Queen's.

3 On a voulu faire de ces deux vers dits par Alceste le dernier mot du Misanthrope. C'est en fait l'avant-dernier : le dernier appartient à Philinthe, qui va chercher à rétablir la relation.

4 Derniers mot de la cinquième lettre de la religieuse portugaise.

5 Voir Patrice Pavis : «Les intertitres, très nombreux et très bavards, dynamisent le récit (...)» (p. 49).

6 Qui n'est pas le plan fixe, comme me l'a appris F. Jost.

7 Patrice Pavis, dans l'article cité plus haut, voit dans l'immobilité une interférence du théâtral : «(...) lorsque l'acteur est longuement filmé, immobile ou répétant la même séquence gestuelle (...), nous sommes (...) dans une temporalité théâtrale» (p. 49).

\section{OUVRAGE CITÉ}

La Fayette, Mme de. La Princesse de Clèves. Paris : Garnier, 1961. 